\title{
TRATAMENTO TÉRMICO E DE NITRETAÇÃO SOB PLASMA DO AÇO INOXIDÁVEL MARTENSÍTICO AISI 420
}

\author{
Carlos Eduardo Pinedo ' \\ Waldemar Alfredo Monteiro ${ }^{2}$
}

\section{Resumo}

O processo de nitretação tem sido usado com algumas limitações para elevar a resposta ao desgaste dos aços inoxidável martensítico tipo AISI 420. Os problemas estão relacionados à combinação entre endurecimento, resistência à corrosão, desempenho em desgaste e dureza do substrato após o tratamento superficial. Este trabalho apresenta a combinação entre diferentes parâmetros de tratamento térmico e superficial de nitretação sob plasma. Diferentes parâmetros de tratamento térmico foram previamente estudados para atingir durezas de substrato entre $38 \mathrm{HCR}$ e $50 \mathrm{HRC}$. As amostras foram nitretadas pelo processo de plasma pulsado. As temperaturas de nitretação variaram entre $480^{\circ} \mathrm{C} \mathrm{e} 560^{\circ} \mathrm{C}$, por tempos entre I hora e 16 horas. A caracterização microestrutural utilizou as técnicas de microscopia óptica e de difração de raios $X$. As durezas do substrato e da superfície foram determinadas pelo método de dureza Rockwell e por microdureza Vickers. A nitretação sob plasma promove um elevado endurecimento, atingindo I.500 HV. Para substratos tratados para $50 \mathrm{HRC}$, a temperatura de nitretação tem uma forte influência no endurecimento. Utilizando uma dureza de partida de $38 \mathrm{HRC}$ o endurecimento superficial atinge seu potencial pleno para todas as temperaturas estudadas. Como consequência, são discutidas diferentes rotas de tratamento térmico e superficial.

Palavras-chave: Aço inoxidável martensítico; Tratamento térmico; Nitretação sob plasma.

\section{INFLUENCE OF HEAT TREATMENT AND PLASMA NITRIDING PARAMETERS ON HARDENING MARTENSITIC STAINLESS STEEL AISI 420}

\begin{abstract}
Nitriding has been used for martensitic stainless steels to increase surface hardness. However, problems may arise related to surface hardness, core hardness, and corrosion wear resistance. This work presents results on the relation between heat treatment and plasma nitriding parameters in order to obtain the best properties combination. Heat treatments were carried out to reach hardness between 38 and 50 HRC. Plasma nitriding were carried out on temperatures ranging from 480 to $560^{\circ} \mathrm{C}$, using times from I to I6 hours. X-ray diffraction, optical microscopy, Rockwell $\mathrm{C}$ hardness and Vickers microhardness techniques were used to characterize the treatments. Results show that plasma nitriding increases surface hardness up to I,500 HV. When substrate is quenched and tempered to $50 \mathrm{HRC}$ the plasma nitriding temperature has a strong influence on hardening behaviour. Increasing nitriding temperature from 480 to $560^{\circ} \mathrm{C}$ substrate hardness drops from 50 to $38 \mathrm{HRC}$. Otherwise, using start tempered and quenched core hardness close to $38 \mathrm{HRC}$ full surface hardening is reached after plasma nitriding and no core hardness drop is verified. As a consequence different routes for heat treatment and plasma nitriding are discussed herein.
\end{abstract}

Key words: Martensitic stainless steel; Heat treatment; Plasma nitriding.

\footnotetext{
' Doutor em Ciências, Diretor Técnico da Heat Tech Tecnologia em Tratamento Térmico e Engenharia de Superfície Ltda, Av. João XXIII, II60, Cep 08830-000, Mogi das Cruzes, SP, Brasil.E-mail: pinedo@heattech.com.br; Professor da Universidade da Mogi das Cruzes. E-mail: pinedo@umc.br ${ }^{2}$ Doutor em Ciências. Professor da Universidade Presbiteriana Mackenzie, CCH, Rua da Consolação, 896, Cep 01302-000, São Paulo, SP, Brasil. Pesquisador do Centro de Ciência dos Materiais e Tecnologia do Instituto de Pesquisas Energéticas e Nucleares. E-mail: walmont@uol.com.br
} 


\section{INTRODUÇÃO}

Os aços inoxidáveis martensíticos são amplamente utilizados em componentes que necessitam de elevada resistência mecânica e à corrosão, por exemplo, em moldes para injeção de polímeros. Os moldes necessitam de aços que atinjam durezas elevadas porque os polímeros de engenharia possuem (i) adição de carga com partículas altamente abrasivas, por exemplo, pelo reforço com fibra de vidro ou nylon ou (ii) são intrinsecamente abrasivos, como os termofixos. Ainda, a resistência à corrosão é necessária na injeção de polímeros clorados, como o PVC, ou no caso de moldes com sistema de câmara quente.

O aço inoxidável martensítico tipo AISI 420 é um dos mais empregados na fabricação de moldes. A possibilidade de endurecimento no tratamento térmico de têmpera e revenimento é uma característica importante desse aço, atingindo durezas de até $50 \mathrm{HRC}$, aproximadamente $500 \mathrm{HV}$. A prática de tratamento térmico sugere uma temperatura de austenitização próxima de $1.025^{\circ} \mathrm{C}$. O revenimento pode ser realizado na faixa de temperatura de $200^{\circ} \mathrm{C}$ ou após o pico de endurecimento secundário, acima de $500^{\circ} \mathrm{C}$. (1) No caso da necessidade de tratamento superficial por nitretação, deve ser utilizado o revenimento em temperatura elevada.

No caso do uso de tratamentos superficiais, é importante pontuar que esses tratamentos não devem comprometer a resistência à corrosão. ${ }^{(2,3)} \mathrm{A}$ nitretação tem sido utilizada com maior frequência para o endurecimento superficial do aço AISI 420, no sentido de elevar a resistência ao desgaste. ${ }^{(2,4)}$ Uma característica particular desse aço é o intenso potencial de endurecimento atingido na nitretação, obtendo-se dureza superior a $1.400 \mathrm{HV} \cdot{ }^{(5,6)}$ Esse forte endurecimento é consequência de uma intensa precipitação de nitretos de cromo na superfície nitretada. Neste trabalho são pesquisados como diferentes parâmetros de tratamento térmico e de nitretação interagem e as possíveis soluções para melhor combiná-los.

\section{MÉTODOS EXPERIMENTAIS}

O material utilizado neste trabalho é aço inoxidável martensítico tipo AISI 420, recebido no estado recozido. A composição química é apresentada na Tabela I. A microestrutura recozida é composta de uma matriz ferrítica com dispersão de carbonetos esferoidizados.

Antes dos tratamentos de nitretação todas as amostras foram previamente temperadas e revenidas. Para a têmpera, as amostras foram austenitizadas a $1.025^{\circ} \mathrm{C}$ e resfriadas em óleo. Os revenimentos foram realizados

Tabela I. Composição química, \% em massa

\begin{tabular}{ccccccccc}
\hline $\mathbf{C}$ & $\mathbf{M n}$ & $\mathbf{S i}$ & $\mathbf{C r}$ & $\mathbf{N i}$ & $\mathbf{V}$ & $\mathbf{N}$ & $\mathbf{P}$ & $\mathbf{S}$ \\
\hline 0,40 & 0,50 & 0,95 & 13,50 & 0,21 & 0,27 & 0,0350 & 0,027 & 0,001 \\
\hline
\end{tabular}

entre $200^{\circ} \mathrm{C}$ e $650^{\circ} \mathrm{C}$. As amostras para nitretação foram previamente revenidas a $520^{\circ} \mathrm{C}$ e $580^{\circ} \mathrm{C}$, objetivando durezas de $50 \mathrm{HRC}$ e $38 \mathrm{HRC}$, respectivamente. Os revenimentos foram duplos de duas horas cada. Todos os tratamentos foram realizados sob vácuo.

Os tratamentos de nitretação sob plasma foram conduzidos em um reator de plasma pulsado-DC, com câmara quente, com potência de $30 \mathrm{kVA}$, marca ELTROPULS GmbH. A remoção do filme passivo de $\mathrm{Cr}_{2} \mathrm{O}_{3}$, fundamental em aços inoxidáveis, porque o filme de óxido atua como uma barreira para a incorporação do nitrogênio, foi realizada por um bombardeamento iônico sob plasma de hidrogênio puro na temperatura de $400^{\circ} \mathrm{C}$ por I hora. A nitretação foi realizada entre $480^{\circ} \mathrm{C}$ e $550^{\circ} \mathrm{C}$ por tempos entre I hora e 16 horas. A mistura gasosa utilizada foi $3 \mathrm{~N}_{2}: 1 \mathrm{H}_{2}$, com pressão de trabalho de $250 \mathrm{~Pa}$ e tensão de $470 \mathrm{~V}$. Durante os tratamentos foram utilizados dois termopares inseridos em furos realizados nas faces laterais das amostras.

A microestrutura da camada nitretada foi observada por microscopia óptica após ataque com Nital $4 \%$. A caracterização das fases presentes foi realizada por difração de raios $X$ em um difratômetro Philips, utilizando radiação $\mathrm{Cu}_{\mathrm{K \alpha}}, \lambda=0,1542 \mathrm{~nm}$, em simetria convencional $\theta / 2 \theta$ de Bragg-Brentano. A avaliação do endurecimento foi realizada por medidas de microdureza Vickers. A dureza do substrato foi avaliada por dureza Rockwell C.

\section{RESULTADOS E DISCUSSÃO}

A Figura I mostra a curva de revenimento obtida na região do endurecimento secundário. A dureza máxima é atingida a $500^{\circ} \mathrm{C}$, como consequência da precipitação de carbonetos complexos. ${ }^{(7)}$ Os resultados mostram que a temperatura de $520^{\circ} \mathrm{C}$ pode ser selecionada para atingir a dureza de 48-50 HRC, preferivelmente indicada para moldes de injeção de polímeros. A temperatura de $580^{\circ} \mathrm{C}$ foi utilizada para atingir dureza de $36 \mathrm{HRC}$ para comparação e definição dos fenômenos relacionados ao endurecimento na nitretação.

A Figura 2 mostra as curvas de endurecimento obtidas após a nitretação sob plasma para amostras previamente revenidas a $520^{\circ} \mathrm{C}$, para a dureza de $49 \mathrm{HRC}$. A nitretação por I hora não é suficiente para atingir o endurecimento máximo. O máximo endurecimento é atingido após a nitretação por 4 horas. Para a nitretação por 16 horas a dureza superficial é inferior à obtida para a nitretação por 4 horas. Comparando-se os resultados para I hora e 4 horas de nitretação, a elevação de dureza observada corresponde ao comportamento esperado. Entretanto, este comportamento não se mantém para o aumento do tempo de 4 horas para 16 horas. Uma vez atingido o endurecimento máximo para 4 horas de trata- 


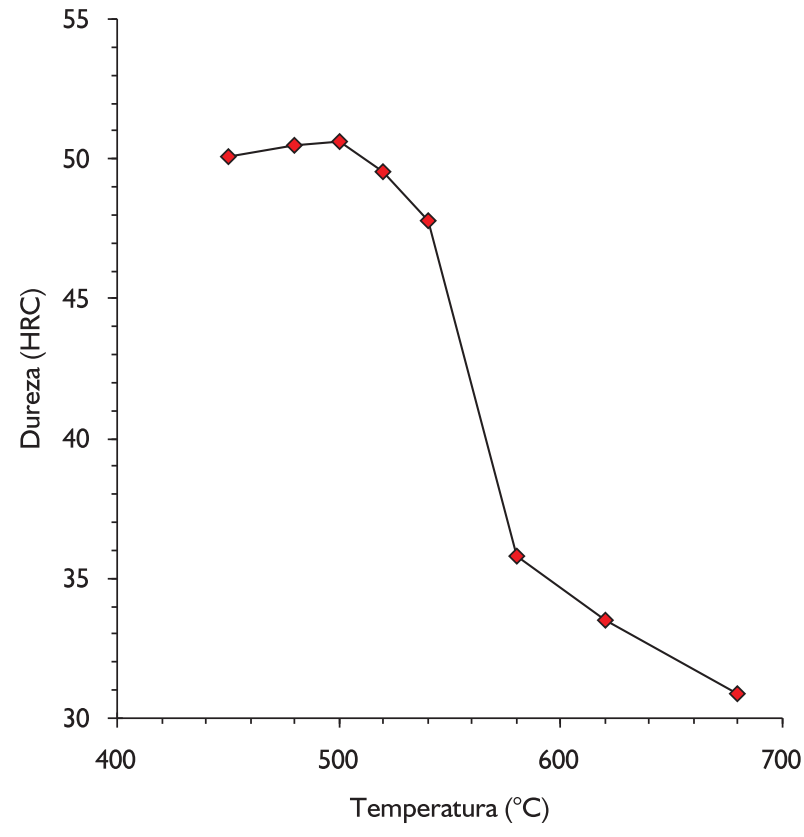

Figura I. Curva de revenimento do aço inoxidável martensítico tipo AISI 420.

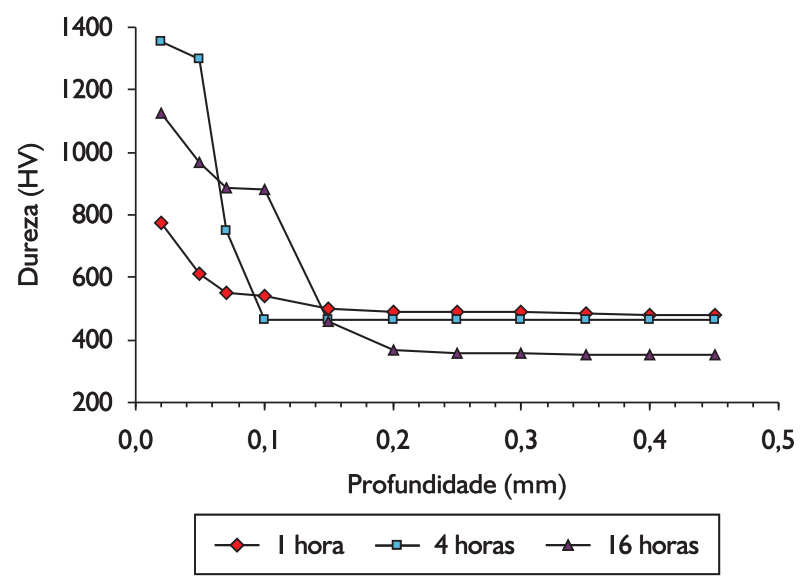

Figura 2. Perfis de dureza determinados após a nitretação sob plasma a $520^{\circ} \mathrm{C}$.

mento seria de se esperar que a dureza máxima tivesse sido mantida, ou até mesmo elevada, com o tempo crescente de 16 horas de nitretação, o que não ocorre por um efeito de super-revenimento.

A dureza do substrato após a nitretação, observada para profundidades superiores a $0,2 \mathrm{~mm}$ mostra uma diminuição de dureza após o tratamento superficial. Para os tempos curtos de tratamento de nitretação - I hora e 4 horas - a dureza do substrato é mantida nos níveis atingidos no revenimento, $470 \mathrm{HV}$. Entretanto, para 16 horas de nitretação observa-se claramente uma queda na dureza do substrato para $35 \mathrm{I} \mathrm{HV}$. Nestas condições, o endurecimento superficial é acompanhado pela queda de dureza do substrato e não pode ser atingido o potencial máximo de endurecimento. Apesar disto, a profundidade de endurecimento aumenta com o aumento no tempo de nitretação.

Estes resultados mostram que a queda de dureza do substrato para tempos prolongados de nitretação a $520^{\circ} \mathrm{C}$ desempenha um papel importante nas características de endurecimento deste aço. Para entender melhor esta queda de dureza durante a nitretação foram realizados tratamentos de envelhecimento por 20 horas em amostras previamente revenidas a $520^{\circ} \mathrm{C}$. Os resultados apresentados na Figura 3 mostram que a dureza obtida no revenimento não se modifica para envelhecimento a $500^{\circ} \mathrm{C}$, mas evidenciam uma forte queda de dureza para temperaturas superiores. Portanto, quando moldes para injeção de polímeros são tratados para 49 HRC (479 HV), o processo de nitretação deve ser controlado para (i) utilizar temperaturas máximas de $500^{\circ} \mathrm{C}$ e (ii) limitando o tempo de nitretação para temperaturas superiores.

A Figura 4 mostra o comportamento de amostras revenidas a $580^{\circ} \mathrm{C}, 620^{\circ} \mathrm{C}$ e $680^{\circ} \mathrm{C}$ e, posteriormente, envelhecidas a $520^{\circ} \mathrm{C}$. Nestas condições não se observa queda de dureza, o que deve permitir uma maior flexibili-

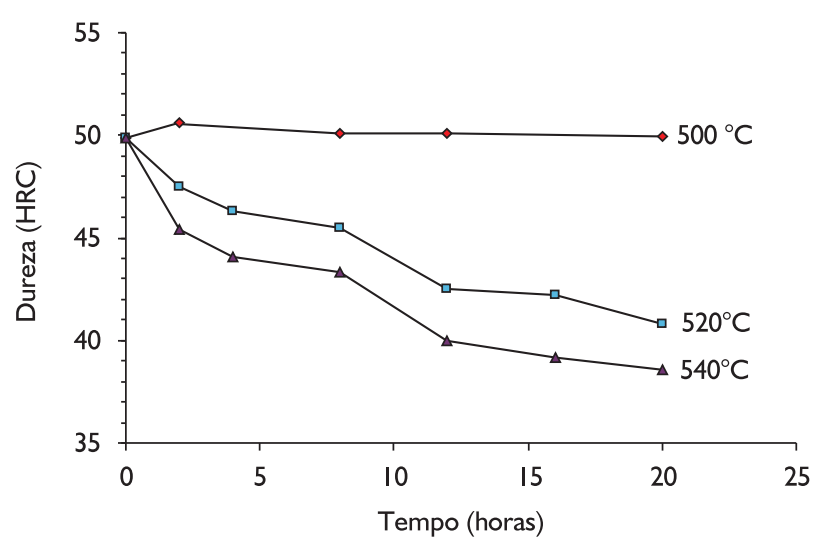

Figura 3. Envelhecimento para amostras revenidas a $520^{\circ} \mathrm{C}$.

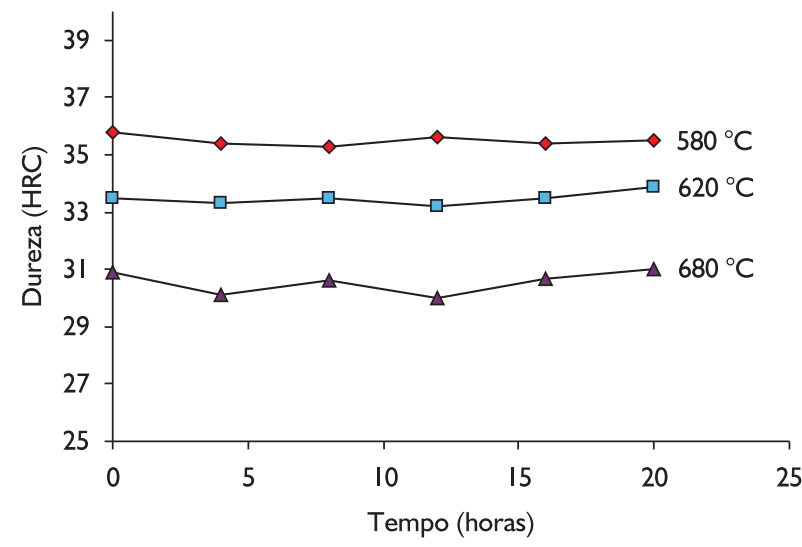

Figura 4. Envelhecimento a $520^{\circ} \mathrm{C}$ para amostras revenidas a $580^{\circ} \mathrm{C}$, $620^{\circ} \mathrm{C}$ e $680^{\circ} \mathrm{C}$ 
dade na seleção de temperaturas e tempos de nitretação. Com o uso de revenimentos em temperaturas elevadas, não deve ser observada a queda de dureza com o tempo de nitretação a $520^{\circ} \mathrm{C}$.

Para definir o mecanismo de endurecimento do aço AISI 420 na ausência do envelhecimento, foram realizados tratamentos de nitretação sob plasma entre $480^{\circ} \mathrm{Ce}$ $560^{\circ} \mathrm{C}$, por 4 horas, em amostras temperadas e revenidas a $580^{\circ} \mathrm{C}$, com substrato na dureza de $35,8 \mathrm{HRC}(360 \mathrm{HV})$. As superfícies nitretadas são observadas na Figura 5. A nitretação a $480^{\circ} \mathrm{C}$ e $560^{\circ} \mathrm{C}$ leva à formação da camada de compostos (C.C.) indicada na Figura 5b, camada branca, incipiente para a menor temperatura. A Zona de Difusão (Z.D.), correspondente à região com precipitação de nitretos de ferro e cromo, aparece fortemente atacada devido à diminuição local da resistência à corrosão decorrente da retirada de cromo em solução sólida para a precipitação dos nitretos tipo $\mathrm{CrN}$. Essa precipitação é verificada pelos resultados de difração de raios $X$ apresentados na Figura 6. A camada nitretada é composta por nitretos do tipo $\gamma^{\prime}-\mathrm{Fe}_{4} \mathrm{~N}, \varepsilon-\mathrm{Fe}_{2-3} \mathrm{~N}$ e $\mathrm{CrN}$.
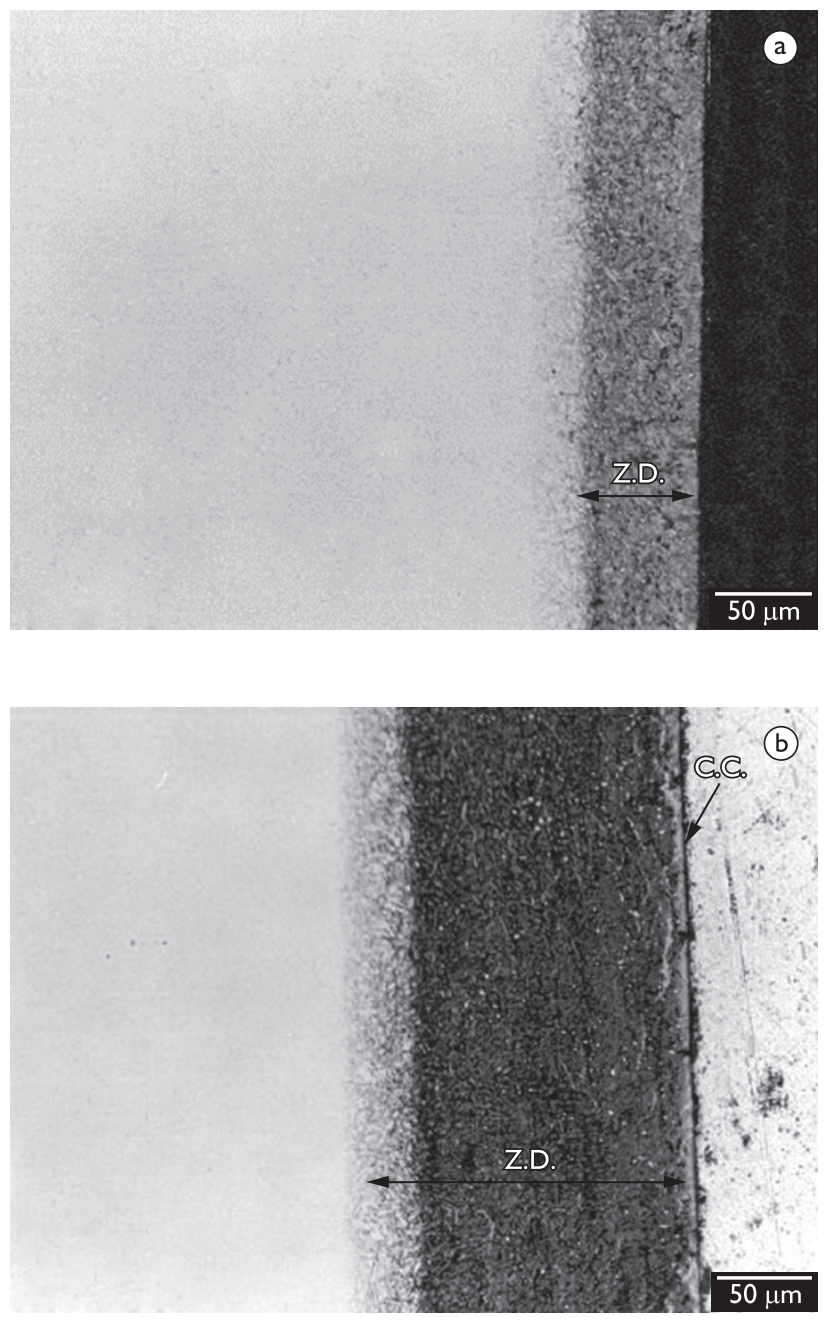

Figura 5. Microestrutura das superfícies nitretadas a $480^{\circ} \mathrm{C}$ (a) e $560^{\circ} \mathrm{C}$ (b). Reagente de Nital $10 \%$.
Os perfis de dureza obtidos nesta condição, apresentados na Figura 7, mostram um intenso potencial de endurecimento para todas as temperaturas de nitretação. O endurecimento é consequência da precipitação fina e homogênea de nitretos na Zona de Difusão ${ }^{(8,9)}$ atingindo o valor máximo de I.560 HV. É importante salientar que, quando o substrato é revenido em temperaturas superiores a de nitretação, não apenas se atinge um máximo de endurecimento com também o endurecimento máximo se mantém por toda a extensão da Zona de Difusão, apresen-

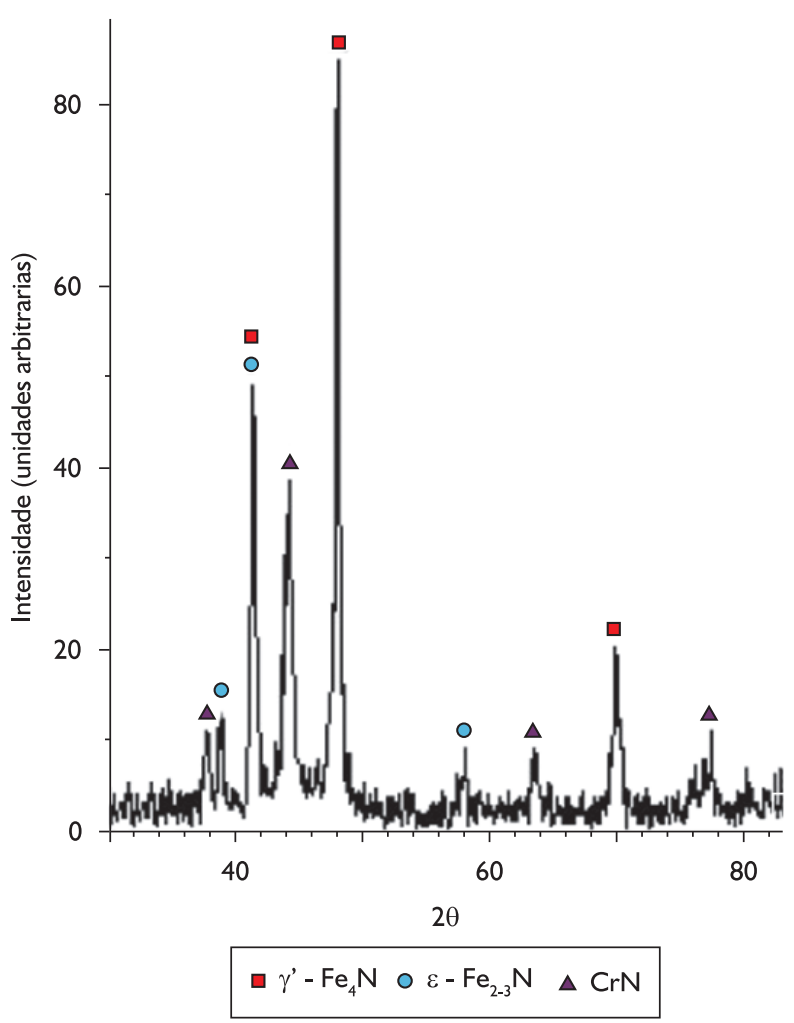

Figura 6. Espectro de difração de raios $X$ na superfície nitretada a $560^{\circ} \mathrm{C}$.

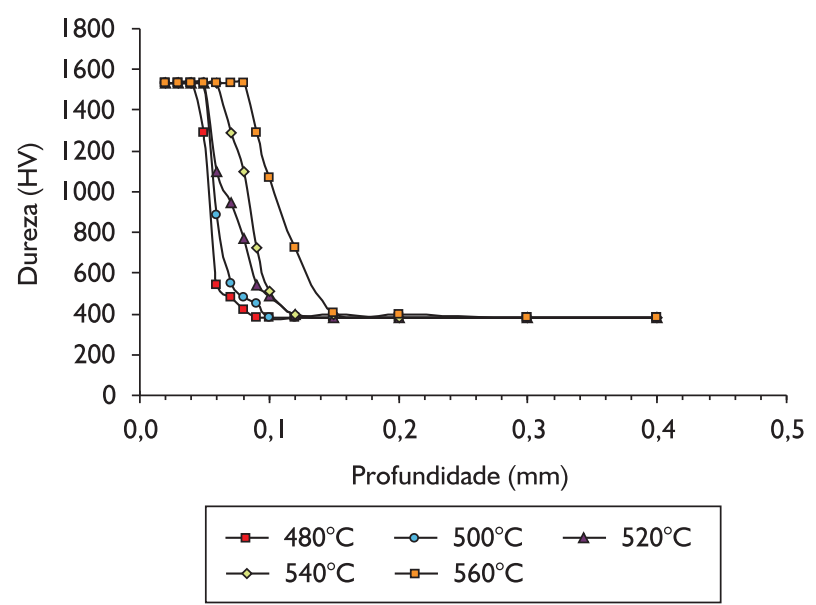

Figura 7. Perfis de endurecimento obtidos após a nitretação por 4 horas em diferentes temperaturas. Substrato revenido a $580^{\circ} \mathrm{C}$. 
tado uma forma plana. A profundidade da camada nitretada aumenta com o aumento do tempo de nitretação. Outra característica importante é a forte queda de dureza após a região de endurecimento máximo, até atingir a dureza do substrato. Este comportamento é consequência da forte interação entre o cromo e o nitrogênio, ${ }^{(10,11)}$ responsável por reações complexas na interface de nitretação. ${ }^{(6)}$

Estes resultados mostram ainda que não ocorre modificação na dureza do substrato nas diferentes temperaturas de nitretação, mantendo-se em 390 HV. Este comportamento é consequência da maior temperatura de revenimento utilizada para substrato no tratamento térmico. ${ }^{(5)}$

\section{CONCLUSÕES}

Os parâmetros de tratamento térmico e nitretação sob plasma podem ser combinados de forma a atingir a máxima resposta ao endurecimento superficial. Considerando a aplicação em moldes para injeção de polímeros, que necessitam de substratos com dureza próxima de 50 HRC, a nitretação sob plasma deve ser realizada em temperaturas inferiores a $520^{\circ} \mathrm{C}$. Para substratos revenidos a $520^{\circ} \mathrm{C}$, com dureza inicial de $500 \mathrm{HV}$, a nitretação promove um intenso endurecimento para tempos de até 4 horas. Aumentando o tempo de nitretação para 16 horas o potencial máximo de endurecimento não é mais atingido por consequência da queda de dureza do substrato por efeito do envelhecimento que ocorre simultaneamente.

Quando a dureza inicial do substrato é diminuída para $36 \mathrm{HRC}$, após revenimento a $580^{\circ} \mathrm{C}$, obtém-se o máximo benefício da nitretação com relação ao envelhecimento. Para as temperaturas de nitretação utilizadas neste trabalho a superfície é composta de uma camada de compostos, camada branca, seguida da Zona de Difusão. A Zona de Difusão apresenta uma interface plana com o substrato, resultado do mecanismo de crescimento no processo de nitretação. A dureza máxima exibe um comportamento plano por toda a extensão da Zona de Difusão e atinge um valor de $\mathrm{I} .560 \mathrm{HV}$.

\section{REFERÊNCIAS}

I UNTERWISER, P. M.; BOYER, H. E.; KUBBS, J. J. Heat treater's guide: standards practices and procedures for steels. Ohio: ASM, 1983

2 Xi, Y.; LIU, D.; HAN, D. Improvement of corrosion and wear resistances of AISI 420 martensitic stainless steel using plasma nitriding at low temperature. Surface and Coatings Technology, v. 202, n. I2, p. 2577-83, Mar. 2008.

3 LI, C. X.; BELL, T. Corrosion properties of plasma nitrided AISI 420 martensitic stainless steel in $3.5 \% \mathrm{NaCl}$ and I\% $\mathrm{NaCl}$ aqueous solution. Corrosion Science, v. 48, n. 8, p. 2046-49, Aug. 2006.

4 TUCKART, W. et al. Sliding behaviour of ion nitrided AISI 420 stainless steel. Surface Engineering, v. 2I, n. 5-6, p. 463-8, Oct. 2005.

5 PINEDO, C. E.; MONTEIRO, W. A. Surface hardening by plasma nitriding on high chromium alloy steel. Journal of Materials Science Letters, v. 20, n. 2, p. 147-9, 200 I.

6 PINEDO, C. E.; MONTEIRO, W. A. On the kinetics of plasma nitriding a martensitic stainless steel type AISI 420. Surface and Coatings Technology, v. I79, n. 2-3, p. I 19-23, Feb. 2004.

7 HONEYCOMBE, R. W. K. Structure and strength of alloy steels. London: Climax Molibdenium, 1973.

8 STAGNO, E. et al. Behaviour of sintered 410 low carbon steel towards ion nitriding. Journal of Alloy Compounds, v. 247, n. I-2, p. 172-9, Jan. 1997.

9 MARKEV, K.; COOPER, C. V.; GIESSEN, B. C. Observation of compound layer with very thin friction coefficient in ion-nitrided martensitic 410 stainless steel. Surface and Coatings Technology, v. 99, n. 3, p. 229-33, Feb. 1998.

I0 JACK, D. H. Nitriding. In: Heat Treatment'73, 1973, London. Proceedings... London: The Metals Society, 1973. p. 39-50.

I I LIGHTFOOT, J.; JACK, D.H. Kinetics of nitriding with and without compound layer formation. In: Heat Treatment'73, 1973, London. Proceedings... London: The Metals Society, 1973. p. 59-65.

Recebido em: $27 / 10 / 2010$

Aceito em: 3 I/03/20 I I 